\title{
ON RECOGNISING CERTAIN ONE RELATION PRESENTATIONS
}

\author{
JAMES $\mathrm{MCCOOL}^{1}$ AND ALFRED PIETROWSKI
}

\begin{abstract}
An algorithm is described to determine, given a finite presentation $\Pi$ of a one relation group $G$ whose commutator subgroup is finitely generated, whether or not $\Pi$ is a one relation presentation.
\end{abstract}

1. Introduction. Let $k, l$ be integers different from $0, \pm 1$. It was shown in [2] that if $r, s$ are integers satisfying $(r, s)=(r, k)=(s, l)=1$, then the group $\left\langle x_{1}, x_{2} ; x_{1}^{k}=x_{2}^{l}\right\rangle$ has presentation

$$
\Pi=\left\langle x_{1}, x_{2} ; x_{1} x_{2}^{l} x_{1}^{-1} x_{2}^{-l}=1, x_{1}^{k} x_{2} x_{1}^{-k} x_{2}^{-1}=1, x_{1}^{s k} x_{2}^{-r l}=1\right\rangle .
$$

In the study of these groups, the problem arises of determining for which values of $r$ and $s$ the presentation $\Pi$ is (in the notation of [2]) a one relation presentation. The object of this note is to prove a result which has as a consequence the fact that, given $k, l, r$ and $s$, it can be determined if the corresponding presentation $\Pi$ is a one relation presentation.

A group $G$ is said to be a one relation group if $G$ has a presentation with a single defining relation. Our result is the following:

THEOREM. Let $G$ be a one relation group with finitely generated commutator subgroup $G^{\prime}$, and let $G$ be given by a presentation

$$
\Pi=\left\langle a_{1}, \cdots, a_{m} ; R_{1}=1, \cdots, R_{n}=1\right\rangle .
$$

Then it can be determined if $\Pi$ is a one relation presentation.

\section{Proof of the Theorem.}

We shall need the following lemma.

LEMma. Let

$$
G=\left\langle b_{1}, b_{2} ; V_{1}=1, \cdots, V_{n}=1\right\rangle,
$$

where the exponent sum of $b_{1}$ in each $V_{i}$ is zero, and $G$ is nonabelian with $G^{\prime}$ finitely generated. Then $\left\{b_{1}, b_{2}\right\}$ is a one relation generating set of $G$ if and only if there exists a positive integer $t$ such that the normal subgroup $N$

Received by the editors February 7, 1972.

AMS 1970 subject classifications. Primary 20F05.

${ }^{1}$ Research supported by a grant from the National Research Council of Canada. 
of $G$ generated by $b_{2}$ is freely generated by the set

$$
\left\{b_{2}, b_{1} b_{2} b_{1}^{-1}, \cdots, b_{1}^{t-1} b_{2} b_{1}^{1-t}\right\} .
$$

Proof. Suppose that such an integer $t$ exists. We put $x_{i}=b_{1}^{i} b_{2} b_{1}^{i-}$ $(1 \leqq i \leqq t-1)$. Since conjugation by $b_{1}$ induces an automorphism of $N$, and the infinite cyclic group $G / N$ is generated by the image of $b_{1}$ under the natural homomorphism from $G$ to $G / N$, it follows that $G$ has a presentation

$$
\begin{aligned}
\left\langle b_{1}, b_{2}, x_{1}, \cdots, x_{t-1} ; b_{1} b_{2} b_{1}^{-1}=x_{1}, \cdots, b_{1} x_{t-2} b_{1}^{-1}\right. & =x_{t-1}, \\
b_{1} x_{t-1} b_{1}^{-1} & \left.=w\left(b_{2}, x_{1}, \cdots, x_{t-1}\right)\right\rangle
\end{aligned}
$$

on its generating set $\left\{b_{1}, b_{2}, x_{1}, \cdots, x_{t-1}\right\}$. Using the obvious Tietze transformations to remove the generators $x_{1}, \cdots, x_{t-1}$, we see that $G$ can be presented on $\left\{b_{1}, b_{2}\right\}$ by

$$
\left\langle b_{1}, b_{2} ; b_{1}^{t} b_{2} b_{1}^{-t}=w\left(b_{2}, b_{1} b_{2} b_{1}^{-1}, \cdots, b_{1}^{t-1} b_{2} b_{1}^{1-t}\right)\right\rangle .
$$

Thus $\left\{b_{1}, b_{2}\right\}$ is a one relation generating set of $G$. The converse follows easily from the proof of Theorem 1 of [3].

To prove the theorem, we first observe that if $m=1$ then $\Pi$ is a one relation presentation, while if $m>2$ then, by Theorem 1 of [3], $\Pi$ is not such a presentation. Thus we can assume that $m=2$.

From $\Pi$ we can obtain, by Theorem 3.5 of [1], a presentation

$$
\Pi_{1}=\left\langle b_{1}, b_{2} ; V_{1}=1, \cdots, V_{n}=1\right\rangle
$$

of $G$ which is $N$-equivalent (in the notation of [2]) to $\Pi$, and such that

$$
V_{i}=b_{i}^{d_{i}} Q_{i} \quad(i=1,2)
$$

and

$$
V_{j}=Q_{j} \quad(2<j \leqq n),
$$

where $d_{i} \geqq 0(i=1,2), d_{2}$ divides $d_{1}$, and $Q_{i}, Q_{j}$ belong to the commutator subgroup of the free group $F\left(b_{1}, b_{2}\right)$ on $\left\{b_{1}, b_{2}\right\}$. Since $\Pi$ and $\Pi_{1}$ are $N$ equivalent, it follows that $\Pi$ is a one relation presentation if and only if the same is true of $\Pi_{1}$.

We now enumerate recursively the presentations of $G$, beginning with $\Pi_{1}$, until we obtain a one relation presentation $\Pi_{2}=\left\langle x_{1}, \cdots, x_{s} ; R=1\right\rangle$, where $s \leqq 2$, and the exponent sum of $x_{1}$ in $R$ is zero if $s=2$. Let $G_{1}, G_{2}$ be the isomorphic copies of $G$ determined by $\Pi_{1}, \Pi_{2}$ respectively. In the process of obtaining $\Pi_{2}$ we can also find words $u_{1}\left(x_{1}, \cdots, x_{s}\right)$, $u_{2}\left(x_{1}, \cdots, x_{s}\right)$ such that the homomorphism $\theta$ from $F\left(b_{1}, b_{2}\right)$ to $F\left(x_{1}, \cdots, x_{s}\right)$ given by $\theta b_{i}=u_{i}(i=1,2)$ induces an isomorphism $\theta: G_{1} \rightarrow G_{2}$. 
Now if $s=1$ and $R$ is nontrivial, then $\Pi_{1}$ is not a one relation presentation, since no single relation on $b_{1}, b_{2}$ can imply that $G_{1}$ is a finite cyclic group, while if $s=1$ and $R$ is trivial then $\Pi_{1}$ is a one relation presentation, since then $G_{1}$ is infinite cyclic and so, from the conditions on the $V_{i}$, we see that $b_{2}=1$. Thus we can assume that $s=2$.

If $G$ is abelian, then, by Problem 21, p. 283 of [1], either $R$ is a primitive element of $F\left(x_{1}, x_{2}\right)$, or $R^{ \pm 1}$ is conjugate in $F\left(x_{1}, x_{2}\right)$ to $x_{1} x_{2} x_{1}^{-1} x_{2}^{-1}$, and in either case $\Pi_{1}$ is a one relation presentation. Now using the algorithm of [4], it can be decided if $R$ is primitive, and it can also be easily decided if $R^{ \pm 1}$ is conjugate to $x_{1} x_{2} x_{1}^{-1} x_{2}^{-1}$. Thus we can assume that $G$ is nonabelian.

Now $G / G^{\prime}$ is infinite, and so the exponent sum of $b_{1}$ in each relator $V_{i}$ of $\Pi_{1}$ must be zero. Let $N_{1}, N_{2}$ be the normal subgroups generated respectively by $b_{2}, x_{2}$ in $G_{1}$ and $G_{2}$. By Corollary 2 of Theorem 1 of [3] the exponent sum of $x_{2}$ in $R$ is nonzero, since the exponent sum of $x_{1}$ in $R$ is zero. Thus if $N$ is a normal subgroup of $G_{2}$ such that $G_{2} / N$ is infinite cyclic, then $x_{2} \in N$ (since $x_{2}$ has finite order in $G_{2} / N$ ) and so $N_{2} \subseteq N$. Now $G_{2} / N_{2}$ is infinite cyclic, and so $N=N_{2}$. Hence $\theta N_{1}=N_{2}$ since $G_{1} / N_{1}$ is infinite cyclic. From the results of [3] we have that $N_{2}$ is a finitely generated free group, and the rank $t$ of $N_{2}$ can be found. Thus $N_{1}$ is free of rank $t$. It follows from the Lemma that $\Pi_{1}$ is a one relation presentation if and only if $\left\{b_{2}, b_{1} b_{2} b_{1}^{-1}, \cdots, b_{1}^{t-1} b_{2} b_{1}^{1-t}\right\}$ is a free generating set of $N_{1}$. This will be the case if and only if $\left\{\theta b_{2}, \theta\left(b_{1} b_{2} b_{1}^{-1}\right), \cdots, \theta\left(b_{1}^{t-1} b_{2} b_{1}^{1-t}\right)\right\}$ is a free generating set of $N_{2}$. This can be decided by expressing the elements of this set in terms of the free generating set $\left\{x_{2}, x_{1} x_{2} x_{1}^{-1}, \cdots, x_{1}^{t-1} x_{2} x_{1}^{1-t}\right\}$ of $N_{2}$, and then applying Nielsen transformations to the resulting set to obtain a Nielsen reduced set. This proves the theorem.

\section{REFERENCES}

1. W. Magnus, A. Karrass and D. Solitar, Combinatorial group theory: Presentations of groups in terms of generators and relations, Pure and Appl. Math., vol. 13, Interscience, New York, 1966. MR 34 \#7617.

2. J. McCool and A. Pietrowski, On free products with amalgamation of two infinite cyclic groups, J. Algebra 18 (1971), 377-383.

3. D. I. Moldavanskii, Certain subgroups of groups with one defining relation, Sibirsk. Mat. Ž. 8 (1967), 1370-1384=Siberian Math. J. 8 (1967), 1039-1048. MR 36 \#3862.

4. J. H. C. Whitehead, On equivalent sets of elements in a free group, Ann. of Math. (2) 37 (1936), 782-800.

Department of Mathematics, University of Toronto, Toronto, Ontario, CANADA 